Relations industrielles

Industrial Relations

\title{
Organizational Psychology, par Bernard M. Bass, Allyn \& Bacon Inc., Boston, January 1965, 459 pages.
}

\section{Hervé Gauthier}

Volume 24, numéro 1, 1969

URI : https://id.erudit.org/iderudit/028011ar

DOI : https://doi.org/10.7202/028011ar

Aller au sommaire du numéro

Éditeur(s)

Département des relations industrielles de l'Université Laval

ISSN

0034-379X (imprimé)

1703-8138 (numérique)

Découvrir la revue

Citer ce compte rendu

Gauthier, H. (1969). Compte rendu de [Organizational Psychology, par Bernard M. Bass, Allyn \& Bacon Inc., Boston, January 1965, 459 pages.] Relations

industrielles / Industrial Relations, 24(1), 228-230.

https://doi.org/10.7202/028011ar

Tous droits réservés @ C Département des relations industrielles de l'Université Laval, 1969
Ce document est protégé par la loi sur le droit d'auteur. L’utilisation des services d'Érudit (y compris la reproduction) est assujettie à sa politique d'utilisation que vous pouvez consulter en ligne.

https://apropos.erudit.org/fr/usagers/politique-dutilisation/ 
ritable théorie dynamique. Sur ces assises, l'auteur tente d'interpréter Keynes en langage Myrdalien, pour finalement enregistrer une contribution personnelle considérable dans la description du cycle économique.

Voici en bref une énumération des grandes lignes de force de la théorie du « cycle des affaires » élaborée dans « Expectations, Investment and Income 》. Chacune des propositions qui suivent est l'objet d'un développement et est étroitement liée à la ou aux précédentes. Le revenu aggrégatif est défini comme la somme des flux, investissement et consommation.

Démonstration de l'hypothèse selon laquelle les changements dans le flux d'investissement sont la cause principale rapprochée et efficiente des changements de revenu. Le flux de l'investissement aggrégatif est fonction des attentes des hommes d'affaires de même que du taux d'intérêt. Comment est déterminé le taux d'intérêt? Le revenu courant obtenu à partir du stock actuel d'équipement s'élèvera si le revenu aggrégatif s'élève.

Suggestion à savoir qu'une hausse inattendue du revenu va à partir de la proposition précédente, causer une revision à la hausse des espoirs de gains réalisables à partir du nouvel équipement projeté ; ce qui influera un accroissement du flux d'investissement. De la même façon et suivant le même processus, la non-réalisation d'un espoir de gain au moyen de l'équipement actuel entraînera une diminution du flux d'investissement.

Suivant la ligne de pensée ébauchée, il est possible à ce moment-ci de dessiner le cycle de causalité suivant: un changement dans les attentes entraîne un changement dans le flux d'investissement lequel entraîne un changement dans le revenu aggrégatif qui lui même conduit à un changement dans les attentes (un laps de temps s'écoulant entre chaque changement et le changement ultérieur auquel il donne naissance).

Description du mécanisme du cycle d'affaire à partir de ces prémisses.

Enfin explication plus profonde du mécanisme du passage d'un revenu aggrégatif croissant à décroissant.

Hervé GAUTHIER
Organizational Psychology, par Bernard M. BASS, Allyn \& Bacon Inc., Boston, January 1965,459 pages.

Jusqu'en 1930, la psychologie industrielle se résumait en une technique attentive aux problèmes personnels et vocationnels. Secondairement, elle s'intéressait aux différences entre les aptitudes individuelles, aux différents degrés d'adaptation et aux facteurs physiques du travail. Cependant, l'attention principale des psychologues industriels est passée progressivement du travailleur manuel, des problèmes associés au travail répétitif, des limitations physiques comme celle de l'éclairage, à l'étude des problèmes des techniciens, des administrateurs, à l'étude des contextes sociaux organisationnels et technologiques qui constituent l'univers fort complexe à l'intérieur duquel doivent oeuvrer ceux-ci.

Ce déplacement du centre d'intérêt a correspondu au déplacement du groupe majeur de travailleurs du primaire, au secondaire jusqu'au tertiaire et a été activé par des événements aussi contraignants que la seconde guerre mondiale et la guerre de Corée: la psychologie industrielle proprement dite inscrit ses premières lignes vers 1940 .

Ces quelques mots d'introduction, l'auteur d' «Organizational Psychology » tient à nous les servir lui-même en apéritif à ce régal toujours recherché qu'est la lecture d'un traité de psychologie industrielle.

La psychologie organisationnelle, c'est l'étude des hommes au travail, affectés par le système hommes, argent et matériaux à l'intérieur duquel ils oeuvrent: de quelle manière sont-ils affectés? C'est aussi l'influence qu'ils exercent sur la conversion de l'investissement énergie humaine, argent et matériel en production de richesse, biens et satisfaction: quelle est la nature et l'amplitude de cette influence? Il y a 3 ou 4 décades, le processus de production était regardé premièrement comme une question d'argent, de matériaux et de travailleurs interchangeables. Aujourd'hui l'étude de la rencontre des travailleurs et des administrateurs comme individus et comme groupes avec l'aspect argent et l'aspect matériaux est privilégié comme agent habilité à permettre la compréhension, la prédictabilité et le contrôle du processus de production. 
L'auteur reprend dans ce volume, dénué d'ésotérismes invincibles, les thèmes classiques de la psychologie industrielle et nous les fait redécouvrir à la lumière des résultats de recherches personnelles. C'est selon ce principe que seront remis sur le métier les phénomènes suivants: La satisfaction au travail peut être considérée comme l'un des objectifs de l'administration et du travailleur; une haute productivité peut en être un autre. Malheureusement l'un n'est pas nécessairement la conséquence de l'autre. Un travail ne satisfera qu'en autant que seront réalisées ou en voie de réalisation les attentes et les aspirations qui $y$ ont attiré et une haute productivité ne suit pas nécessairement la réalisation de ces aspirations individuelles.

Les bénéfices matériels, les bonnes conditions de travail et le travail qu'on considère comme un défi continuel sont quelques-uns des facteurs indépendants qui additionnent à la satisfaction au travail. Quelles sont les conditions qui accroissent ou réduisent la matérialisation et la puissance de ces générateurs de satisfaction?

Un même travail devra, selon l'individu qui l'effectue, être apte à réaliser autant d'attentes spécifiques qu'il y a de différences entre chacun sur la conception de ce qui est important. De plus pour que puisse être possible la perception de l'intérêt du travail confié, celui-ci devra être adapté aux capacités du travailleur: si le travailleur exige trop, il y aura frustration de réussite, s'il n'exige pas assez, l'ennui envahira l'atmosphère de l'atelier. L'individu pourra surmonter ces sentiments dans la fuite ou le combat.

Les superviseurs de groupe à «morale $\gg$ élevée diffèrent de ceux des groupes à «morale » basse en ce qu'ils se penchent plus fréquemment sur le travail de leurs subordonnés, en ce qu'ils acceptent volontiers la discussion de problèmes communs avec ces mêmes subordonnés; en ce qu'ils initient des discussions de groupe; en ce qu'ils tiennent ceux-là au courant des dernières informations. L'attitude des superviseurs est un facteur passablement important de l'atmosphère qui règne dans l'usine et de l'attitude que prendront en réponse les supervisés.

La cohésion des membres d'un groupe influence fortement l'ardeur et la satisfaction au travail. Un groupe a atteint la maturité lorsque ses membres s'acceptent mutuellement, se fient les uns aux autres, communiquent ouvertement et sont capables d'identifier leurs buts propres à ceux du groupe. La grandeur du groupe est une caractéristique dans laquelle réside la graine de sa dégénérescence. Le processus de prise de décision des groupes sera d'autant plus efficace que les composants connaîtront et accepteront leurs rôles respectifs, qu'ils seront coopératifs plutôt que compétitifs, tolérants les uns envers les autres et orientés vers la correction mutuelle des défauts de performance.

Les organisations industrielles se présentent selon une variété presqu'incroyable de granceurs, sont mus par une infinie diversité de buts spécifiques. Il est cependant possible de retrouver dans chacune des structures en place un aspect formel et informel: l'aspect formel, c'est l'organisme, les livres de règlements, les habitudes qui ont force de loi, les conventions collectives; l'aspect informel c'est les relations qui se forment entre les travailleurs indépendamment du travail qu'ils exécutent et qui sont la résultante du fait qu'un contremaître peut être simple membre d'une organisation sociale dont son subordonné au travail est vice-président. L'analyse de l'organisation industrielle à la poursuite d'un but doit faire interagir d'une façon réaliste ces deux aspects.

Comment se propagent l'information et les communications à l'intérieur de l'entreprise? Quelles sont les structures de transmission des politiques et des ordres de la haute direction? Comment les suggestions, questions et griefs gravissent-ils l'échelle de la supervision? Le bloquage et la filtration font se répandre des rumeurs. On réalise très peu souvent la grande quantité du flux d'information qui se déplace horizontalement.

Les conflits à l'intérieur de la structure formelle de l'entreprise se manifestent avec une certaine consistance. A différents niveaux de la hiérarchie correspondront vraisemblablement souvent des désaccords sur les buts de l'organisation et la contrainte à exercer pour les atteindre. Les différences de statut confuses ou non associées à des réelles différences de compétence et d'estime reposent sur un lit de salpêtre. La méfiance interpersonnelle fera souvent de ces conflits un combat à finir d'où il ne résul- 
tera pour l'organisation aucun progrès sensible: le vainqueur détient le championnat jusqu'au match revanche. Des efforts sont quotidiennement faits pour que la prise de décision soit un mécanisme finement rationnel. Trois étapes constituent essentiellement le processus de solution des problèmes: la perception, la recherche de solutions originales et l'évaluation de ces solutions. Les caractéristiques du problème et du chercheur vont facilement influencer les résultats de chacune de ces étapes d'où la difficulté d'éliminer complètement le biais dû aux attitudes de base et aux motivations de ceux qui affrontent ces problèmes.

\section{Hervé GAUTHIER}

Peach Ceri, West Indian Migration to Britain, Oxford University Press, London 1968.

Le travail présenté dans cet ouvrage est un essai d'analyse et d'interprétation des mouvements migratoires entre les Indes Occidentales et la Grande-Bretagne.

L'analyse se fonde sur des statistiques récentes mais incomplètes. Elle tente de décrire globalement et quantitativement les groupes d'émigrants, de définir leur destination exacte et de retrouver les lieux de leur installation. L'analyse conduit à une interprétation des causes et conséquences de ces mouvements migratoires. Les causes sont simples et peu définies; elles ne permettent donc pas l'élaboration d'un modèle d'analyse pour d'autres situations de même genre. Les conséquences sont étudiées trop rapidement et par rapport à une situation trop particulière; elles empêchent donc de prévoir, sur une échelle universelle, les suites de phénomènes semblables.

En somme, cet ouvrage présente un intérêt certain, mais très particulier. Il n'invite pas à des études plus approfondies et ne permet pas une théorisation certes nécessaire dans ce domaine.

\section{René PARENTEAU}

Essays in Labour History, by Asa Briggs and John Saville, Papermac 191, Macmillan 1967, 364 pages.

Cet ouvrage a été préparé en collaboration et il est dédié à la mémoire de ce grand historien du travail que fut
G.D.H. Cole, professeur à Oxford en son temps, et qui pourrait être aussi bien cité comme économiste que sociologue ou politicologue.

Les éditeurs présentent d'abord un certain nombre de textes à caractère biographique rédigés par des personnes qui ont bien connu le professeur.

Mais l'essentiel de l'ouvrage est constitué de neuf essais en histoire du travail sous la signature d'autant d'historiens qui d'une façon ou d'une autre ont suivi les traces du maître. L'ensemble de ces essais présente un vaste tableau de l'histoire du travail, notamment en Grande-Bretagne, au cours du dix-neuvième siècle.

Il $\mathrm{y}$ est question tout aussi bien $\mathrm{du}$ concept de classe du début de ce siècle que de la participation de certains éléments du mouvement ouvrier anglais à la première Internationale.

On y trouve aussi des textes sur les circonstances qui ont entouré l'affaire Taff Vale, sur les conditions de travail en terme d'usages, de salaires et de charge de travail au XIXe siècle, et quelques autres.

Briggs et Saville mettent ainsi un précieux instrument de travail à la disposition de ceux qui s'intéressent à cette période importante de l'histoire du travail en Grande-Bretagne.

\section{Jean BERNIER}

The Nature of Price Theory, by H.H. Liebhapsky, Revised Edition, The Dorsey Press, Homewood. Illinois, August 1968, 643 pages.

Malgré les prétentions de l'auteur à l'effet que la lecture de son excellent manuel exige du lecteur qu'il sache seulement additionner, soustraire, multiplier et diviser, il faut bien avouer que son livre ne se lit pas comme un roman-policier et qu'il exige davantage.

Ecrit surtout à l'intention des étudiants sous-gradués, il suppose de la part du lecteur, sinon un entraînement mathématique minimum, du moins un certain esprit mathématique et une certaine habileté à traduire dans ce langage et celui de la géométrie les relations fondamentales que l'on peut abstraire de la réalité économique. 